\title{
The Impact of COVID-19 Infection on Labor and Delivery, Newborn Nursery, and Neonatal Intensive Care Unit: Prospective Observational Data from a Single Hospital System
} \author{
M. Cecilia DiPentima, MD ${ }^{1}$ Andrew Schenkman, MD ${ }^{1,2,3}$

\footnotetext{
${ }^{1}$ Department of Pediatrics, Morristown Medical Center, Morristown, New Jersey

${ }^{2}$ Department of Clinical and Transnational Research, Biomedical Research Institute of New Jersey, Cedar Knolls, New Jersey

${ }^{3}$ MidAtlantic Neonatal Associates, Morristown, New Jersey

${ }^{4}$ Department of Obstetrics and Gynecology, Morristown Medical Center, Morristown, New Jersey

${ }^{5}$ Department of Maternal Fetal Medicine, Morristown Medical Center, Morristown, New Jersey
}

Ian Griffin, MD ${ }^{1,2,3}$ Farah Benarba, MD $\quad$ Caryn Peters, MSN ${ }^{1,3}$ Yinka Oyelese, MD ${ }^{5}$

Tom Murphy, MD ${ }^{1,3}$ Diana Contreras, MD ${ }^{4}$ Christina Gagliardo, MD ${ }^{1}$ Eberechi Nwaobasi-Iwuh, MD ${ }^{1}$

Am J Perinatol 2020;37:1022-1030.

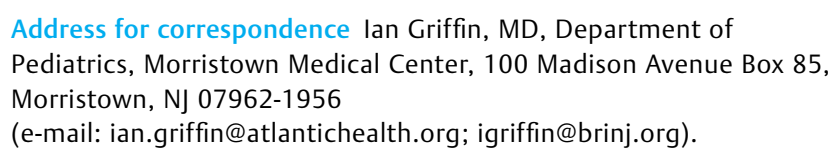

Address for correspondence lan Griffin, MD, Department of Pediatrics, Morristown Medical Center, 100 Madison Avenue Box 85, Morristown, NJ 07962-1956 (e-mail: ian.griffin@atlantichealth.org; igriffin@brinj.org).

\begin{abstract}
Keywords

- COVID-19

- SARS-CoV-2

- vertical transmission

- mother-infant dyad

- horizontal transmission

Objective Since its emergence in late 2019, severe acute respiratory syndromecoronavirus-2 (SARS-CoV-2), the novel coronavirus that causes novel coronavirus disease 2019 (COVID-19), has spread globally. Within the United States, some of the most affected regions have been New York, and Northern New Jersey. Our objective is to describe the impact of COVID-19 in a large delivery service in Northern New Jersey, including its effects on labor and delivery (L\&D), the newborn nursery, and the neonatal intensive care unit (NICU).

Materials and Methods Between April 21, 2020 and May 5, 2020, a total of 78 mothers (3.6\% of deliveries) were identified by screening history or examination to either be COVID19 positive or possible positives (persons under investigation). Of the mothers who were tested after admission to L\&D, 28\% tested positive for SARS-CoV-2.

Discussion Isolation between mother and infant was recommended in 62 cases, either because the mother was positive for SARS-CoV-2 or because the test was still pending. Fiftyfour families (87\%) agreed to isolation and separation. The majority of infants, 51 (94\%), were initially isolated on the newborn nursery. Six needed NICU admission. No infants had clinical evidence of symptomatic COVID-19 infection. Fourteen infants whose mothers were positive for SARS-CoV-2, and who had been separated from the mother at birth were tested for SARS-CoV-2 postnatally. All were negative.

Results COVID-19 posed a significant burden to mothers, infants, and staff over the 5week study period. The yield from screening mothers for COVID-19 on L\&D was high. Most families accepted the need for postnatal isolation and separation of mother and newborn.
\end{abstract}

received

May 18,2020

accepted after revision

May 21,2020

published online

June 13,2020
Copyright $\odot 2020$ by Thieme Medical

Publishers, Inc., 333 Seventh Avenue, New York, NY 10001, USA Tel: $+1(212) 760-0888$.
DOI https://doi.org/ 10.1055/s-0040-1713416. ISSN 0735-1631. 
Conclusion Our study suggests that the transmission of SARS-CoV-2 from mother to her fetus/newborn seems to be uncommon if appropriate separation measures are performed at birth.

Key Points

- The yield of targeted testing for SARS-CoV-2, on mothers on Labor and Delivery is high.

- Agreement to separation of mothers and infants to reduce transmission of SARS-CoV-2 was high.

- The incidence of symptomatic COVID-19 in newborns is low, if appropriate separation occurs at birth.

Since its emergence in late 2019 until early May 2020, severe acute respiratory syndrome-coronavirus-2 (SARS$\mathrm{CoV}-2$ ), the novel coronavirus that causes novel coronavirus disease 2019 (COVID-19), has infected more than 3,680,000 people, almost one-third of them in the United States $(1,204,000) .{ }^{1}$ Within the United States, the two most affected states have been New York $(312,000$ cases or 1,652 / 100,000 population) and New Jersey $(130,000$ cases or $1,461 / 100,000$ population). ${ }^{2}$

Within New Jersey, the counties closest to New York have been most affected by SARS-CoV-2. On May 5, 2020, the Northern counties of Passaic and Union reported rates of infection of 2,740 and 2,420/100,000 population respectively, while Southern counties such as Cape May (399 cases/ 100,000 population) and Atlantic (448 cases/100, 000 population) had been relatively less affected. ${ }^{3}$

Despite significant increases in both incidence and prevalence, little is known about COVID-19 in the perinatal period. ${ }^{4,5}$ Limited data suggest pregnancy may not be a risk factor for severe disease. ${ }^{6-8}$ Though most case series suggest vertical transmission is relatively unlikely, there is a case report of a newborn infant with early positive testing suggestive of vertical transmission in utero. ${ }^{9}$

The objectives of the study are to describe the effects of COVID-19 on the obstetric and neonatal population in an area with a high incidence of SAR-CoV-2 infection.

\section{Materials and Methods}

\section{Setting}

The study was conducted at two hospitals within the Atlantic Health System (AHS) in New Jersey. The system has two large obstetric services one at Morristown Medical Center, and one at Overlook Medical Center. Morristown Medical Center (MMC), a tertiary referral center with a level-4 neonatal intensive care unit (NICU), and 4,597 deliveries in 2019, is located within Morris Country, NJ. As of May 5, 2020, Morris county reported a prevalence of 1,154 cases of COVID-19 per 100,000 population, and 96 deaths from COVID-19 per 100,000 populations. ${ }^{3}$ Overlook Medical Center (OMC) is in Union County, $\mathrm{NJ}$, which is one of the most heavily affected counties in NJ reporting 2,420 cases per 100,000 population and 139 deaths per 100,000 population from COVID-19 as of May 5, 20. ${ }^{3}$ OMC had 2,587 deliveries in 2019.

\section{Patient Population}

The study population consisted of maternal-infant dyads whose mothers were identified to be either COVID-19 positive or persons under investigation (PUI) before their admission to labor and delivery (L\&D) or at any time before their discharge.

Women who presented to L\&D but did not deliver infants were excluded, as were pregnant women who presented to the gynecology service, or women pregnant with viable fetuses who were being cared for outside L\&D, unless they delivered. Neonates who were readmitted from home were also excluded.

\section{Duration}

The study started on March 31, 20 when the first wave of obstetric patients who were COVID-19 positive or PUIs began to be identified within AHS and continued for 5 weeks, ending on May 5, 2020.

\section{Design}

This was a prospective observational cohort study. Patients were identified from logs maintained on L\&D, newborn nursery, and the NICU. Data were extracted from the electronic medical record. The Institutional Review Board (IRB) of Atlantic Health System exempted the study from IRB review and for the need for patient consent.

\section{Management of Obstetric Patients}

The patient and her support person underwent a temperature check prior to entering the hospital. On admission to L\&D, expectant mothers and their support person were asked if they, any household members, or any other close contact had been evaluated for COVID-19, or had had fever, cough, breathing difficulties, sore throat, headache, muscle aches, chills, or loss of taste or smell. Any affirmative answer to these questions made the mother a PUI.

Obstetric patients who were COVID-19 positive or PUIs were cared for in a designated suite of single-person airborne infection isolation (AIIRs) negative pressure rooms separate from the main L\&D unit through delivery and the postpartum period, while awaiting testing for COVID-19 or if they had tested positive for COVID-19. All care providers wore recommended personal protective equipment (PPE) and visitors were not allowed, except for the designated support person. 
Efforts were made to limit the number of caregivers who interacted with the mother, the frequency of interactions (especially invasive ones), as much as far as was consistent with high quality care of the mother and fetus. A single designated support person wearing a surgical mask was allowed during L\&D and postpartum hospitalization. Mothers were also required to wear masks. ${ }^{10}$

Maternal PUI status or COVID-19 positivity was considered an indication for a prenatal neonatal consult. The prenatal consult explained the recommendation for separation and isolation of the infants, consistent with current Center for Disease Control and Prevention and American Academy of Pediatrics guidelines. ${ }^{11-13}$ If families were not able to agree to this, their informed choice was respected and documented in the medical record.

Delayed umbilical care clamping was allowed for all infants if the Obstetric team felt that the infant was sufficiently stable. The infant was shown to the mother following delivery. Neither holding of the infant, nor skin-to-skin contact between the mother and infant were permitted.

If mothers tested negative for SARS-CoV-2, they were transferred back to a regular maternity room.

\section{Resuscitation of Infants of PUls and Mothers who Were COVID-19 Positive}

Maternal PUI or COVID-19 positivity was considered an indication for neonatal attendance at delivery. A single neonatal provider (neonatologist or neonatal nurse practitioner) was present in the delivery or operating room, wearing appropriate PPE (including an N95 mask). The rest of the neonatal team waited outside the room with PPE available. If the infant required more than routine resuscitation (drying, stimulation, and suctioning), additional members of the neonatal team were called in to assist once they had donned their PPE. Otherwise they waited outside the room. After resuscitation, the infant was shown to the family, maintaining a 6 -foot distance from the mother, and transferred to a clean isolette outside the room. The rest of the neonatal team took the infant to an isolation room in the newborn nursery or NICU as appropriate, while the designated provider within the room doffed their PPE.

Neither maternal COVID-19 positivity nor maternal PUI status was a contraindication to any resuscitative measures (including cardiopulmonary resuscitation, continuous positive airway pressure [CPAP], intubation, or ventilation) that the neonatal team felt to be required for the infants.

\section{Postnatal Care of Infants of PUls and Mothers who Were COVID-19 Positive}

Healthy asymptomatic neonates who were over 35 weeks of gestation and over 2-kg birth weight were isolated in a designated room in the newborn nursery using droplet precautions. Caregivers wore appropriate PPE including surgical masks, face shields, gowns, gloves, and shoe covers when caring for these infants, and infants remained in isolettes. The designated room was not equipped with negative pressure. Infants were bathed as soon as possible after birth. Visitors were not allowed. Early discharge of these infants was allowed if medically appropriate. ${ }^{14}$ Brief periods of removal from the isolette were permitted at the discretion of the care team to allow for care or procedures such as circumcision to occur

If separation of the infant was refused by a family, asymptomatic neonates were allowed to room in with their mother. Physical separation of six feet between symptomatic individuals and infants, and the use mechanical barriers (e.g., curtains) were strongly encouraged. The infant was cared for in an isolette. Routine newborn care was provided by asymptomatic healthy caregivers wearing a mask, and after performing careful hand hygiene. When direct contact between an affected mother or a maternal PUI, and the infant was necessary, the mother was required to wear a mask and perform hand hygiene prior to contact. Skin-to-skin contact and breastfeeding were not recommended.

Human milk feeds were encouraged and mothers were provided electronic breast pumps, and lactation support to provide mother's own milk that could be fed to the infant during their period of isolation, and to allow direct breastfeeding once the infant and/or mother was cleared from isolation.

For infants less than 35 weeks of gestation or weighing less than $2 \mathrm{~kg}$ at birth were admitted to the NICU, as were infants from L\&D or the newborn nursery (NBN) whose medical condition required NICU admission. Two separate, designated spaces were established in the NICU for newborn infants under investigation depending on whether they did or did not have respiratory distress. Infants with respiratory distress who required aerosolizing therapies (e.g., CPAP) or interventions (e.g., nasopharyngeal suctioning) were placed in AIIR rooms under airborne and contact isolation. Designated staff wearing PPE (consistent with airborne precautions and including single-use N95 respirator, face shield, and gowns) provided care in that space. Infants without respiratory distress but who required NICU level of care (e.g., for evaluation and management of hypoglycemia) were placed in a non-negative pressure isolation room on droplet and contact precautions. Staff wearing PPE (surgical masks and disposable gowns) provided care in that space. All NICU infants were cared for in isolettes.

Parent visitation was not allowed for newborn infants in the NICU isolation rooms who were born to mothers with COVID-19 or who were PUIs.

Newborn infants were cleared from isolation precautions depending on the results of the mother's test and the clinical scenario. Newborn infants whose mother's tested negative for COVID-19 were cleared from isolation as soon as the test resulted. If a mother tested positive for COVID-19 and newborn infants had been immediately separated at birth from their mother, neonatal isolation precautions were suspended after two negative polymerase chain reaction (PCR)-based nasopharyngeal swab tests, performed at 48 hours and at 5 days of life, respectively. Asymptomatic neonates born to SARS-CoV-2 mothers who were not separated at birth (either because of refusal of separation or because of postpartum diagnosis), remain on isolation precautions for 14 days and after two negative reverse transcriptase PCR (RT-PCR)-based nasopharyngeal swab tests, performed at 5 and 10 days of life. 


\section{Postdischarge Recommendations for Mother and Neonates}

If the mother's COVID-19 testing was still pending at the time of discharge, they were advised to continue isolation and separation until the test had resulted negative. If the initial test for COVID-19 was positive, families were advised that after discharge the infant should be carried wherever possible by a healthy asymptomatic adult who was without COVID-19, did not have contact with a COVID-19 patient, and was without symptoms of COVID-19 for the previous 14 days. Ideally, that person would be aged less than 65 years and free of risk factors for severe COVID-19 disease (obesity, heart disease, pulmonary disease, or compromised immune function). If no such person was available, the infant was to be cared for by a parent who should maintain a distance of 6-feet from the infant as much as possible, and carry out proper hand hygiene and wear a mask when caring for the infant. If only one parent was symptomatic, it was recommended that care should be provided by the asymptomatic parent. The symptomatic parent should remain 6-feet from the infant as much as possible, and wear a mask and perform hand hygiene when interacting with the infant.

If the mother tested negative for COVID-19 before discharge, normal newborn care, without restrictions, was recommended.

\section{Laboratory Diagnosis}

Testing of admitted pregnant women and newborns was performed using flocked plastic nasopharyngeal swabs placed in $3 \mathrm{~mL}$ of transport media. At the onset of the pandemic, testing of SARS-CoV-2 was available only through the New Jersey Department of Health, using the Center for Disease Control and Prevention platform. On March 17, AHS began sending inpatient nasopharyngeal specimens to BioReference Laboratories (Elmwood Park, NJ).On April 10, inhouse "rapid" RT-PCR (Xpert Xpress SARS-CoV-2, Cepheid, CA) became available but with limited capacity.

\section{Analysis}

Descriptive statistics (mean, percentage, and counts) were calculated for the different outcome. Between group differences were assessed using Student's $t$-test for continuous normal data, and Fisher's exact test or Chi-square test for categorical data.

To simplify the graphical representation of some of the data, the entire period of observation was divided into five subperiods, week 1 (March 31,-April 6, 2020), week 2 (April 7-April 13, 2020), week 3 (April 14-April 20, 2020), and week 4 (April 21-27, 2020) and week 5 (April 28, 2020 onwards). Analysis was performed using Stat 14.2 for Macintosh (StataCorp, College Station, TX). A p-value less than 0.05 was considered significant.

\section{Results}

\section{Mothers Identified as COVID-19 Positive or PUls}

Eight mothers were known to be COVID-19 positive at the time of $L \& D$ admission due to a previous positive test performed for symptoms consistent with COVID-19 infec-

\begin{tabular}{|c|c|}
\hline Symptom $^{\mathrm{a}}$ & $n(\%)$ \\
\hline Fever & 31 (39) \\
\hline Respiratory symptoms & $22(28)$ \\
\hline Contact $^{\mathrm{b}}$ & $16(20)$ \\
\hline Diarrhea & $12(15)$ \\
\hline General malaise & $10(13)$ \\
\hline Atypical PIH or HELLP syndrome & $3(4)$ \\
\hline Other ${ }^{c}$ & $2(3)$ \\
\hline Unclear & $2(3)$ \\
\hline
\end{tabular}

Abbreviations: COVID-19, novel corona virus disease 2019; HELPP, hemolysis, elevated liver enzymes low platelet count syndrome; $\mathrm{PIH}$, pregnancy induced hypertension; PUI, persons under investigation. Note: Totals do not sum to $100 \%$ as many mothers had more than one symptom.

a 61 patients had one of the seven symptoms, 5 had two symptoms, 7 had three symptoms, 2 had four symptoms, 1 had five symptoms, and no patients had six or more symptoms.

${ }^{b}$ Contact with a COVID-19 positive individual, a PUI, or person with symptoms suggestive of COVID-19 infection, or pyrexia in the support person present on labor and delivery.

'Anosmia $(n=1)$, hypoxemia $(n=1)$.

tions. An additional 70 mothers were identified after admission and were designated as PUIs. Factors that prompted designation of a mother as a PUI included fever, respiratory symptoms (cough, shortness of breath, and upper respiratory symptoms), or contact with a potentially infected person (this includes the detection of fever in the spouse or support person of the mother present during labor; -Table 1).

Of the eight mothers known to be COVID-19 positive before L\&D admission, three had been tested within the previous 10 days. They were not retested, and they were treated as presumptive positives, and separation from their infants was recommended. Five mothers had tested positive more than 14 days before admission to L\&D. Four were retested of whom three remained positive, and one was negative. However, the negative result was not available until after the mother was delivered so the mother was treated as presumed positive pending the result of the test, so the infant was initially isolated until the mother's negative test was resulted. The final mother was not retested but was cleared by the adult infectious diseases service and that infant was not isolated.

Of the 70 mothers identified as PUIs without previous results, 1 refused consent for testing (and was treated as a presumptive positive), 69 mothers were tested of whom 19 were positive ( $28 \%$ of tested PUIs; - Fig. 1 ).

The final status of the 78 mothers in the study was, therefore, that 26 patients (33\%) were either positive or presumed positive for COVID-19, and 52 were either negative or presumed negative.

During the study period, 713 mothers delivered at MMC and OMC. COVID-19-positive mothers and PUIs comprised $11 \%$ of deliveries. Overall, $3.6 \%$ of mothers who delivered during the study period were diagnosed as being COVID-19 positive. 


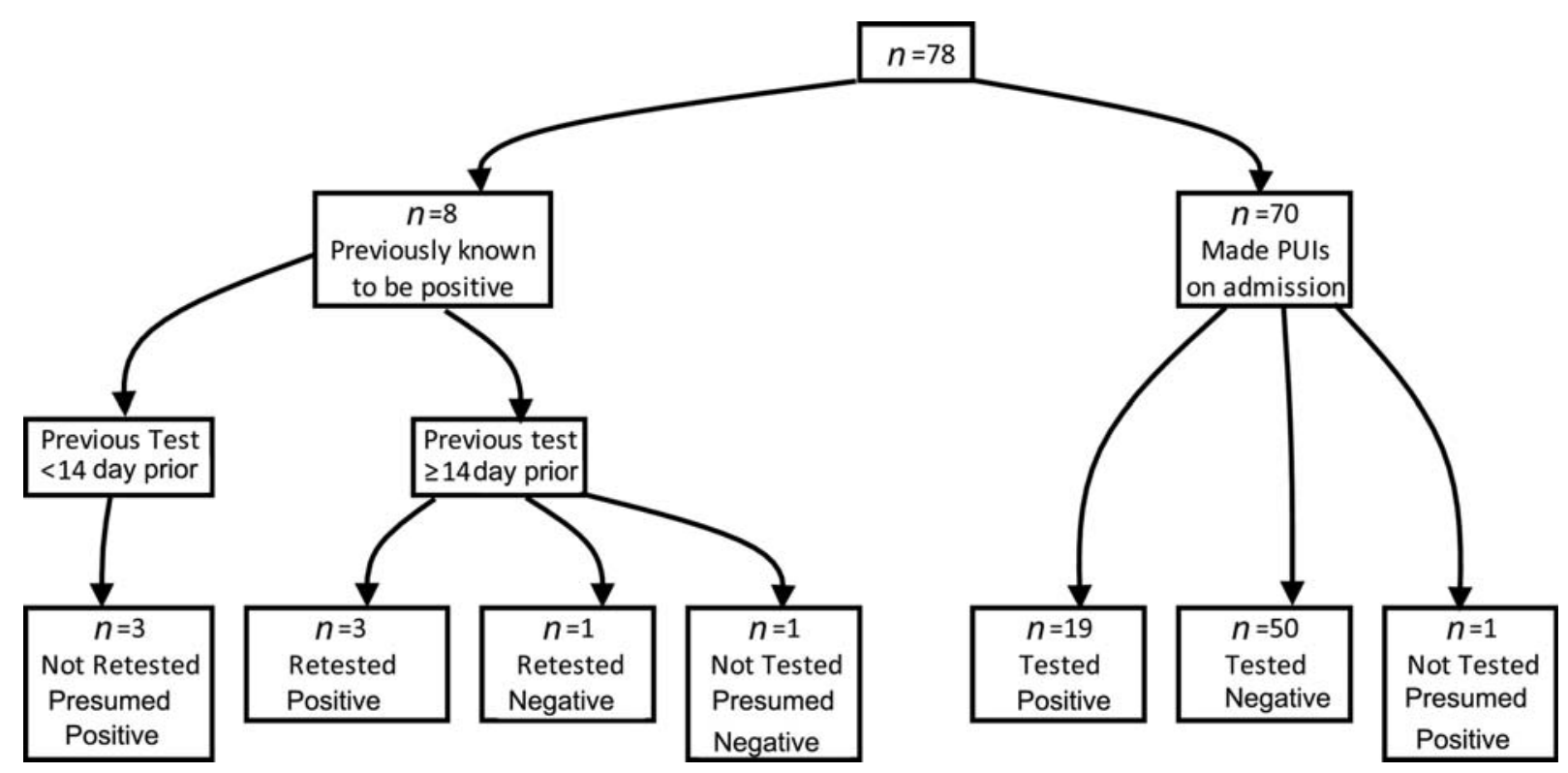

Fig. 1 Schematic showing the timing and results of tests for mothers who were tested prior to L\&D admission and those tested after being identified as PUls after admission. Of the 79 mothers, 22 were COVID-19 positive, and 4 were presumed to be positive. COVID-19, novel corona virus disease 2019; L\&D, labor and delivery; PUI, persons under investigation.

In the early stages of the study, COVID-19 testing was only carried at New Jersey Department of Health laboratories with limited availability and turn-around time was variable that could take up to 5 days to result. Subsequent access to commercial and in-house testing allowed results to be available in a more timely manner (-Fig. 2). Over the 5week period the number of mothers who had COVID-19 tests resulted and available to guide management of them and their infants before delivery increased from $7 \%$ in week 1 to $73 \%$ in week 5 ( - Fig. 2 ).

\section{Infants of COVID-19-Positive Mothers}

Twenty-five mothers had final results of COVID-19 testing available before they delivered; of them, 15 (60\%) were negative and the infants did not need isolation. One infant was cleared from isolation by the adult infectious disease

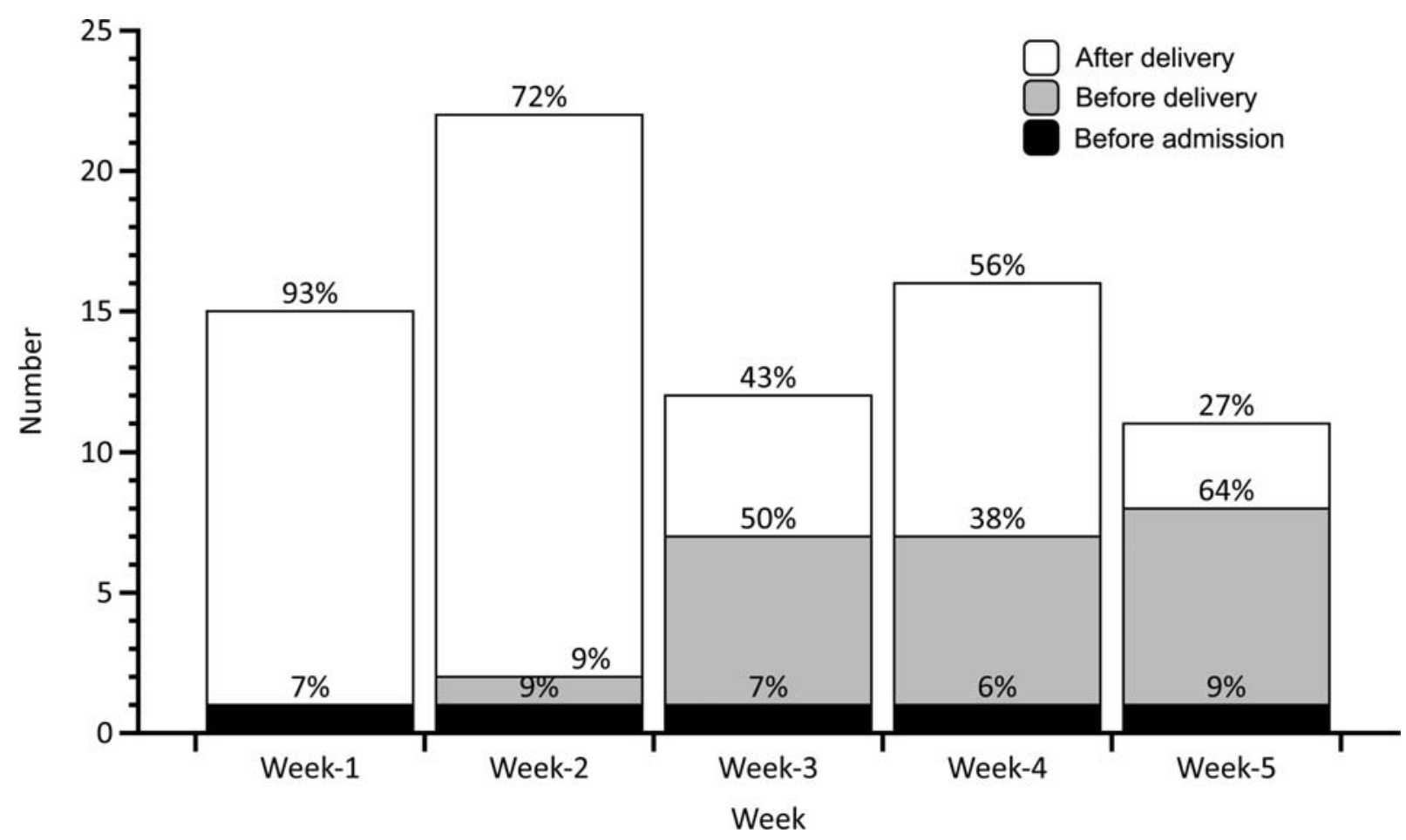

Fig. 2 Cumulative bar graph of the number of maternal COVID-19 test that were results before admission to L\&D, after admission (but before delivery of the infant), and after delivery of the infant. The percentages for each week are shown above the respective bar. COVID-19, novel corona virus disease 2019; L\&D, labor and delivery. 


\begin{tabular}{|c|c|c|c|c|}
\hline & $\begin{array}{l}\text { Mother COVID-19 } \\
\text { positive/unknown }\end{array}$ & $\begin{array}{l}\text { Mother COVID-19 } \\
\text { negative }\end{array}$ & $p$-Value & All \\
\hline$n$ & 27 & 35 & - & 62 \\
\hline Gender (male:female) & $13: 14$ & $14: 21$ & 0.68 & $27: 35$ \\
\hline Gestational age (wk) & $38.9 \pm 1.5$ & $39.3 \pm 1.3$ & 0.22 & $39.0 \pm 1.4$ \\
\hline Birth weight $(\mathrm{g})$ & $3317 \pm 501$ & $3371 \pm 458$ & 0.66 & $3,348 \pm 474$ \\
\hline Length at birth $(\mathrm{cm})$ & $50.9 \pm 2.91$ & $51.4 \pm 1.87$ & 0.41 & $51.1 \pm 2.47$ \\
\hline Head circumference at birth (cm) & $34.0 \pm 1.56$ & $34.2 \pm 1.66$ & 0.52 & $34.1 \pm 1.58$ \\
\hline Delivery & & & 0.13 & \\
\hline Vaginal & 17 & 25 & & 42 \\
\hline Repeat cesarean section & 5 & 2 & & 7 \\
\hline Primary cesarean section & 3 & 1 & & 4 \\
\hline Emergent cesarean section & 2 & 7 & & 9 \\
\hline
\end{tabular}

Abbreviation: COVID-19, novel corona virus disease 2019.

anknown refers to two mothers who were presumed positive but did not have positive RT-PCR results, one mother refused consent for testing, and one mother's sample was insufficient for analysis.

service without retesting as the mother had been positive for COVID-19 24 days prior to admission but was asymptomatic. Isolation was therefore recommended for 62 infants. Of those 62 patients, isolation and separation at birth was accepted by 54 families (87\%) and refused by 8 . Final maternal COVID-19 testing was positive in 25 (40\%), unknown in 2 (one not tested and sample insufficient for one), and negative in 35 (56\%).

The demographic information for the 62 infants in whom separation was recommended is shown in - Table 2.

Of the 54 infants isolated, 51 were initially transferred to the newborn nursery. Their clinical course was generally unremarkable, and they had no complications that were felt to be related to COVID-19 infection ( - Table 3 ). All 54 infants were discharged home to follow-up with their own pediatrician.

Three infants were initially admitted to the NICU for isolation, and a further three isolated infants were transferred from NBN. None of these admissions or transfers were felt to be due to COVID-19. Nor did any of these infants suffer complications that were felt to be related to COVID-19 ( - Table 4).

Once isolated (either on the NICU or NBN) infants remained isolated until maternal COVID-19 testing was negative. If mother's COVID-19 testing was negative, isolation was discontinued as soon as it was reported (-Table 5).

\section{COVID-19 Status of Infants}

Fifteen infants had their SARS-CoV-2 RT-PCR testing performed from a nasopharyngeal swab taken after 24 hours of life. Most (14, 93\%) were performed in neonates whose mothers were confirmed COVID-19 positive. Fourteen of the tests were negative, one was insufficient for analysis. No infant tested positive for COVID-19.

\section{Number of Infants Isolated}

The number of infants requiring isolation peaked on April 10, 2020 (-Fig. 3). The subsequent decline is presumably due to

\begin{tabular}{|c|c|c|c|c|}
\hline No. & $\begin{array}{l}\text { Gestational } \\
\text { age (wk) }\end{array}$ & Complication & $\begin{array}{l}\text { Maternal } \\
\text { COVID-19 } \\
\text { status }\end{array}$ & $\begin{array}{l}\text { Related to } \\
\text { COVID-19 }\end{array}$ \\
\hline 1 & 39 & Developed respiratory distress and required transfer to the NICU & Negative & No \\
\hline 2 & 40 & LGA IDM, hypoglycemia refractory to glucose gel. Transferred to NICU & Negative & No \\
\hline 3 & 39 & Failed hearing screen (unilateral) & Negative & No \\
\hline 4 & 37 & Sepsis work-up, no antibiotics ${ }^{\mathrm{a}}$ & Negative & No \\
\hline 5 & 40 & Failed hearing screen (unilateral) & Positive & No \\
\hline 6 & 37 & Sepsis workup, no antibiotics ${ }^{\mathrm{a}}$ & Negative & No \\
\hline 7 & 41 & SGA, hypoglycemia refractory to glucose gel. Transferred to NICU & Negative & No \\
\hline 8 & 36 & Hypoglycemia requiring one glucose gel & Positive & No \\
\hline
\end{tabular}

Abbreviations: COVID-19, novel corona virus disease 2019; LGA, large for gestational age; NICU, neonatal intensive care unit; SGA, small for gestational age; IDM, Infant of a diabetic mother.

abased on the Kaiser-Permanente neonatal sepsis calculator. ${ }^{17}$ 


\begin{tabular}{|c|c|c|c|c|}
\hline No. & $\begin{array}{l}\text { Gestational } \\
\text { age (wk) }\end{array}$ & Reason for admission/transfer & $\begin{array}{l}\text { Maternal } \\
\text { COVID-19 status }\end{array}$ & $\begin{array}{l}\text { Related to } \\
\text { COVID-19 }\end{array}$ \\
\hline 1 & 39 & Transferred from NBN with respiratory distress & Negative & No \\
\hline 2 & 35 & Admitted from L\&D with prematurity, and respiratory distress & Positive & No \\
\hline 3 & 40 & Transferred from NBN with hypoglycemia refractory to glucose gel. & Negative & No \\
\hline 4 & 34 & Admitted from L\&D with prematurity, and respiratory distress & Negative & No \\
\hline 5 & 40 & $\begin{array}{l}\text { Admitted from L\&D with hypoxic ischemic } \\
\text { encephalopathy requiring whole body cooling }\end{array}$ & Positive & No \\
\hline 6 & 41 & Transferred from NBN with hypoglycemia refractory to glucose gel & Negative & No \\
\hline
\end{tabular}

Abbreviations: COVID-19, novel corona virus disease 2019; L\&D, labor and delivery; NBN, newborn nursery; NICU, neonatal intensive care unit.

Table 5 Results of maternal testing, and timing of results, and implications for length of isolation for the infants, for the 54 infants who were isolated

\begin{tabular}{|lll|}
\hline $\begin{array}{l}\text { Time mother's } \\
\text { test resulted }\end{array}$ & $\begin{array}{l}\text { Positive } \\
\text { tests }\end{array}$ & $\begin{array}{l}\text { Negative } \\
\text { tests }\end{array}$ \\
Before delivery & $9^{\mathrm{a}}$ & - \\
Before day of discharge & $9^{\mathrm{a}}$ & $15^{\mathrm{b}}$ \\
On day of discharge & $2^{\mathrm{a}}$ & $3^{\mathrm{b}}$ \\
After discharge & $5^{\mathrm{a}}$ & $10^{\mathrm{a}}$ \\
\hline
\end{tabular}

${ }^{a}$ These infants (and an additional infant who was not tested but whose mother was presumed to be positive) required isolation for their entire hospital stay and beyond $(n=36)$.

${ }^{b}$ These infants $(n=18)$ required isolations for part or most of the hospital stay. a decreasing incidence of new COVID-19 cases within the Northern New Jersey region, and the decreased test turnaround time of testing that allowed an increased number of negative results to be identified prior to delivery ( - Fig. 2 ), obviating the need for isolation when mothers were COVID19 negative.

\section{Discussion}

We examined the effect on our maternity, delivery, and neonatal services over the 5 -week study period that corresponded to the peak in new cases, and hospitalizations from COVID-19 in Northern New Jersey. During that period, $11 \%$ of the mothers who delivered in our facilities were either COVID-19 positive or PUIs. Ultimately, $3.6 \%$ of mothers were diagnosed as COVID-19 positive. At the same time (ending May 5, 2020) the cumulative incidence of COVID-

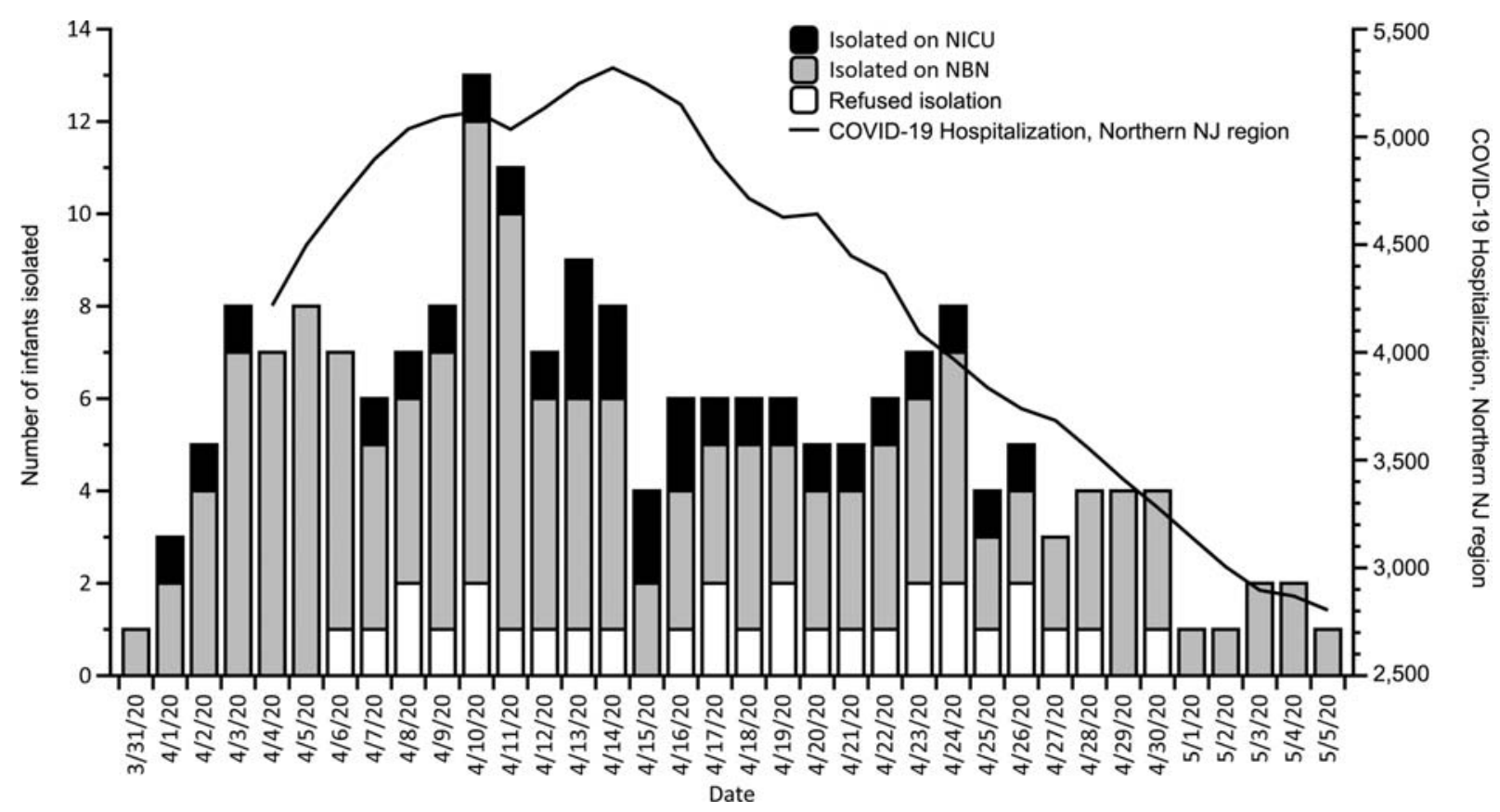

Fig. 3 Number of infants needing isolation on the NICU or NBN, or refusing isolation, by study day. Also shown is the total number of patients hospitalized with COVID-19 in the Northern New Jersey (NJ) region (comprising Bergen, Essex, Hudson, Morris, Passaic, Sussex, Union, and Warren Counties) on the same day (data was not available for days prior to April 4, 2020). ${ }^{3}$ COVID-19, novel corona virus disease 2019; NBN, newborn nursery; NICU, neonatal intensive care unit. 
19 infection was 2,420 per 100,000 population in Union county, home of OMC, and 1,460 per 100,000 population in Morris county, home of MMC, or 2.4 and $1.5 \%$ respectively. ${ }^{3}$ Whether, the higher rate observed in delivering mothers was due to an increased susceptibility, or due to higher rates of testing is not clear. It seems likely, however, that some of our mothers would not have been tested for COVID-19 unless they had been screened on admission to L\&D.

Our screening criteria to identify mothers presenting to L\&D as PUIs was relatively broad. Despite this, the yield from testing of mothers identified as PUIs on L\&D was high, with $28 \%$ (95\% confidence interval [CI]: $17.5-39.6 \%$ ) testing positive for COVID-19. This high rate of positivity would seem to support the use of the screening methods we used as a useful method for identifying COVID-19-positive mothers on L\&D at least while the community incidence of COVID-19 is high.

Our approach to the management of infants born to PUIs or COVID-19-positive mothers was deliberatively conservative, but in line with the recommendations of the Center for Disease Control, and Prevention ${ }^{12}$ the American Academy of Pediatrics, ${ }^{13}$ and others. ${ }^{15}$ Given the risk of horizontal transmission between an infected mother and her infant, and the unknown consequences of neonatal infection, we strongly advised separation between COVID-19-positive mothers (or PUIs pending results) to limit the risk to the infant. We recognize that this was very difficult for families, and that others have recommended less stringent measures for mothers who are only mildly symptomatic or asymptomatic, ${ }^{16}$ but given the uncertainties related to the likelihood of, and consequences of, postnatal horizontal transmission to the newborn infant, we felt this was the only prudent approach. Despite the emotional and practical difficulties attendant on separation of the mother and infant, most families (87\%) accepted this advice. If families were unable to accept this advice despite careful counseling, their opinion was respected, and the infant was allowed in room with the mother.

Most infants born to COVID-19-positive mothers or PUIs were carried for on the NBN. Their clinical course was generally unremarkable, and no adverse effects or complications were felt to be attributable to COVID-19 infection. Similarly, those infants who required admission to the NICU did not seem to have disease related to COVID-19, and most of them (or their mothers) were subsequently found to be SARS-CoV-2 negative.

Although symptomatic infection with SARS-CoV-2 seems to be unlikely in our newborn population, we are limited in our ability to comment on asymptomatic neonatal infection. We did not routinely test neonates for SARS-CoV-2, instead we first waited for the results of maternal testing. This decision was one of distributive justice, for much of the study period COVID-19 testing was in limited availability and the turn-around time could be as much as 5 days. We felt that it was not typically appropriate to test infants before mother's result were available as follows:

- If a mother tested negative for SARS-CoV-2, an asymptomatic newborn would be very unlikely to test positive, if maternal results were true false negative results. Testing the infant before the mother's result was available would have used a test that could have better been used elsewhere, and may have delayed the ending of separation (if this became dependent on the infant's test being negative).

- If a mother tested positive for SARS-CoV-2 and appropriate separation measures had been performed, an asymptomatic newborn would be unlikely to test positive at birth. In that case, separation between mother and infant would need to continue until the mother was cleared of COVID-19 infection.

The only instance where testing of newborns at the time of birth might be more helpful in understanding the clinical course of neonatal infection is when positive neonates are not separated from their infected mothers. If mother and infant were COVID-19 positive, the impact of ongoing separation is not known. Based on the high transmissibility of SARS-CoV-2, separation of the newborn from known infected caregivers would be advisable to prevent ongoing exposure. Thus, the same recommendations for infected and uninfected infants would apply.

The moral dimensions of this decision were complex (weighing individual benefit against distributive justice) but ultimately felt we had struck the correct balance. As testing for COVID-19 becomes more available and results become more quickly available, we expect that analysis to change.

The burden on staff in caring for the mothers and infants should not be underestimated. Among infants, the peak number of isolated infants preceded the peak in hospitalization in Northern NJ. The decline in number of infants that need to be isolated was a result of (1) the decreasing incidence of COIVD-19 in the population, and (2) the increasing availability of "rapid" testing that was able to clear mothers prior to discharge.

\section{Strengths and Limitations}

Our study has several strengths, such as prospective data collection, and single-health care system with uniform policies and approaches at both study sites. Our main limitation was that not all infants born to COVID-19-positive mothers were tested for COVID-19 early in the pandemic. However, our study emphasizes the need to allocate limited resources to areas of clinical care that require critical decision making, and provides encouragement that the likelihood of symptomatic vertical transmission from mother to fetus is low.

Conflict of Interest

None declared.

\section{References}

1 John Hopkins University. COVID-19 Dashboard by the Center for Systems Science and Engineering (CSSE) at Johns Hopkins University (JHU). Available at: https://coronavirus.jhu.edu/map. html. Accessed May 5, 2020 
2 Han GK. COVID-19 Live Interactive Dashboard (v3). Available at: https://datastudio.google.com/reporting/f56febd8-5c42-4191bcea-87a3396f4508/page/GQFJB. Accessed May 5, 2020

3 State of New Jersey Department of Health. New Jersey COVID-19 Dashboard. Available at: https://www.nj.gov/health/cd/topics/ covid2019_dashboard.shtml. Accessed May 5, 2020

4 Mimouni F, Lakshminrusimha S, Pearlman SA, Raju T, Gallagher PG, Mendlovic J. Perinatal aspects on the covid-19 pandemic: a practical resource for perinatal-neonatal specialists. J Perinatol 2020;40(05):820-826

5 Kimberlin DW, Stagno S. Can SARS-CoV-2 infection be acquired in utero?: more definitive evidence is needed JAMA 2020 (e-pub ahead of print). Doi: $10.1001 /$ jama.2020.4868

6 Li N, Han L, Peng M, et al. Maternal and neonatal outcomes of pregnant women with COVID-19 pneumonia: a case-control study. Clin Infect Dis 2020 (e-pub ahead of print). Doi: 10.1093/cid/ciaa352

7 Chen H, Guo J, Wang C, et al. Clinical characteristics and intrauterine vertical transmission potential of COVID-19 infection in nine pregnant women: a retrospective review of medical records. Lancet 2020;395(10226):809-815

8 Schwartz DA. An analysis of 38 pregnant women with COVID-19, their newborn infants, and maternal-fetal transmission of SARSCoV-2: maternal coronavirus infections and pregnancy outcomes. Arch Pathol Lab Med 2020 (e-pub ahead of print). Doi: 10.5858/ arpa.2020-0901-SA

9 Alzamora MC, Paredes T, Caceres D, Webb CM, Valdez LM, La Rosa M. Severe COVID-19 during pregnancy and possible vertical transmission. Am J Perinatol 2020 (e-pub ahead of print) . Doi: 10.1055/s-0040-1710050

10 Society for Maternal Fetal Medicine and Society for Obstetric Anesthesia and Perinatology. Labor and Delivery COVID-19 Con- siderations. Available at: https://s3.amazonaws.com/cdn.smfm.org/media/2277/SMFM-SOAP_COVID_LD_Considerations_3-2720_(final)_PDF.pdf. Accessed May 10, 2020

11 CDC. Considerations for Inpatient Obstetric Healthcare Settings. Available at: https://www.cdc.gov/coronavirus/2019-ncov/hcp/ inpatient-obstetric-healthcare-guidance.html. Accessed May 5, 2020

12 CDC. Care for Breastfeeding Women: Interim Guidance on Breastfeeding and Breast Milk Feeds in the Context of COVID-19. Available at: https://www.cdc.gov/coronavirus/2019-ncov/hcp/ care-for-breastfeeding-women.html. Accessed May 6, 2020

13 Puopolo KM, Kimberlin DW. Management of Infants Born to Mothers with COVID-19. Available at: https://downloads.aap.org/AAP/PDF/COVID\%2019\%20Initial\%20Newborn\%20Guidance. pdf. Accessed May 4, 2020

14 American Academy of Pediatrics. Committee on Fetus and Newborn. Hospital stay for healthy term newborns. Pediatrics 2010; 125(02):405-409

15 Kuzniewicz MW, Puopolo KM, Fischer A, et al. A quantitative, riskbased approach to the management of neonatal early-onset sepsis. JAMA Pediatr 2017;171(04):365-371

16 De Rose DU, Piersigilli F, Ronchetti MP, et al; Study Group of Neonatal Infectious Diseases of The Italian Society of Neonatology (SIN). Novel Coronavirus disease (COVID-19) in newborns and infants: what we know so far. Ital J Pediatr 2020;46 (01):56

17 Davanzo R, Moro G, Sandri F, Agosti M, Moretti C, Mosca F. Breastfeeding and coronavirus disease-2019: Ad interim indications of the Italian Society of Neonatology endorsed by the Union of European Neonatal \& Perinatal Societies. Matern Child Nutr 2020 (e-pub ahead of print). Doi: 10.1111/mcn.13010 